\title{
The Ni's Solution for Neutron Star and Outward Oriented Gravitational Attraction in Its Interior
}

\author{
Luboš Neslušan \\ Astronomical Institute, Slovak Academy of Sciences, Tatranska Lomnica, Slovakia \\ Email: ne@ta3.sk
}

Received 2 October 2015; accepted 6 December 2015; published 9 December 2015

Copyright (C) 2015 by author and Scientific Research Publishing Inc.

This work is licensed under the Creative Commons Attribution International License (CC BY). http://creativecommons.org/licenses/by/4.0/

c) (i) Open Access

\begin{abstract}
In 2011, Jun Ni published the solution of the Tolman-Oppenheimer-Volkoff equations describing the structure of stable neutron stars, which implies that 1) there is no upper mass limit of these objects, 2) their outer physical surface is always situated above the corresponding event horizon, and 3) the object is a hollow sphere with the inner physical surface and cavity inside. In our paper, we propose to "purify" the general relativity, as the geometrical theory, from the concept of mass. If we get rid of the concept of mass and Newtonian-type potential, then we obtain such the behavior of gravity which results in the above mentioned stable Ni's object. It is farther pointed out that the distribution of matter, which is observed as spherically symmetric by the observer in its center, is not longer observed as spherically symmetric by an observer aside the center in a curved spacetime of general relativity. This fact implies, in contrast to the Newtonian physics, the nonzero and outward oriented gravitational attraction of upper layers of star. Ni considered positive energy density and pressure. In addition, gravity had everywhere attractive character. No "exotic" assumption was made. Hence, there is no reason why his concept of hollow sphere should not be applicable to the models of real objects.
\end{abstract}

\section{Keywords}

Relativistic Compact Objects, Neutron Stars, General Relativity, Ni’s Solution

\section{Introduction}

In their famous work published in 1939, Oppenheimer and Volkoff [1] concluded that there is no solution for a stable configuration of dead star, without an internal source of energy, if the mass of the star exceeds a certain critical limit, which was later named by them. In the subsequent work, it was demonstrated that the star has to 
collapse below its event horizon [2].

The conclusion about the non-existence of the stable configuration of very massive stars and the necessity of their collapse to become the black holes has been regarded as valid more than three quarters of century and is hard to believe that something could ever bring a doubt about this claim. In 2011, Chinese researcher Jun Ni [3] however published a solution, which forces us to deal again with the question on the stable configuration of very massive stars. Ni attempted to reproduce the creation of the Oppenheimer-Volkoff model of neutron star. He proceeded in the same way as the original authors of the model, except of the starting point of the numerical integration of the differential equations, which are relevant to the model. While Oppenheimer and Volkoff started the integration in the center of the object, $\mathrm{Ni}$ started it in a finite star-centric distance.

The inward proceeded part of the Ni's integration always ended with zero pressure and energy density in a finite star-centric distance, i.e. he obtained a model with an inner physical surface of the object. In addition, he concluded that there is no upper-mass limit. And, the outer physical surface appears to be always situated above the corresponding event horizon.

In this paper, we attempt to analyze the reason why Ni was successful in the creation of the stable model for very massive compact object. Clearly, the Ni's result evokes some fundamental questions on the principles and postulates within the general relativity, especially those, which were originally established within the Newtonian physics and appeared in general relativity after their formal generalization.

\section{TOV Model of Neutron Star}

\subsection{The Original Basic Equations}

The first model of the star that spent all its storage of nuclear fuel was worked out for the spherically symmetric, non-rotating objects by Oppenheimer and Volkoff [1]. They used the description of gravity almost simultaneously published by Tolman [4]. Hence, this kind of model is named as the Tolman-Oppenheimer-Volkoff or TOV model. This model includes the equation of state (EoS, hereinafter) published by Chandrasekhar [5] who derived this EoS considering some ideas from the previous work by Landau [6]. The Chandrasekhar's EoS describes the state of a pure neutron, extremely cold and, thus, degenerated gas. Hence, the objects of this kind started to be named as neutron stars (NSs), although there are known, today, also their models consisting of a more complex, not only neutron, matter.

To describe the gravity, Tolman [4] considered the Einstein field equations (EFEs) [7] [8]. He simplified these equations for the case of spherical symmetry. (The EFEs for the spherical symmetry were already published by Eddington [9] earlier. Tolman specified more some details.)

In our paper, we also aim to construct the TOV simple model of non-rotating and, therefore, spherically symmetric NS. So, we use the already mentioned form of the EFEs for the spherical symmetry explicitly given by Eddington [9] and Tolman [4]. We remind these equations:

$$
\begin{aligned}
& \kappa P=\mathrm{e}^{-\lambda}\left(\frac{v^{\prime}}{r}+\frac{1}{r^{2}}\right)-\frac{1}{r^{2}}, \\
& \kappa P=\mathrm{e}^{-\lambda}\left(\frac{1}{2} \nu^{\prime \prime}-\frac{1}{4} \lambda^{\prime} v^{\prime}+\frac{1}{4} v^{\prime 2}+\frac{1}{2 r} v^{\prime}-\frac{1}{2 r} \lambda^{\prime}\right), \\
& \kappa E=\mathrm{e}^{-\lambda}\left(\frac{\lambda^{\prime}}{r}-\frac{1}{r^{2}}\right)+\frac{1}{r^{2}} .
\end{aligned}
$$

The meaning of used symbols is following. $P$ is the pressure, $E$ is the energy density, and $\lambda$ and $v$ are the auxiliary functions related to the components of metric tensor $g_{r r}$ and $g_{t t}$ as $g_{r r}=-\mathrm{e}^{\lambda}$ and $g_{t t}=\mathrm{e}^{v}$. In the static case, $\lambda$ and $v$ are only the functions of the star-centric radial distance $r$. The prime indicates the derivative with respect to $r$. Because of a better transparency we need in our explanations, the equations are given in the form assuming the usage of the SI units. In this system of units, constant $\kappa=8 \pi G / c^{4}$, where $G$ is the gravitational constant and $c$ is the speed of light. Using Equations (1)-(3), it is possible to derive the equation for the gradient of pressure balancing the gravity in the form

$$
\frac{\mathrm{d} P}{\mathrm{~d} r}=-\frac{E+P}{2} v^{\prime}
$$


Oppenheimer and Volkoff [1] replaced function $\lambda$ by another auxiliary function, $u$, defined by

$$
u=\frac{1}{2} r\left(1-\mathrm{e}^{-\lambda}\right) \text {. }
$$

Subsequently, the gradient of pressure can be given in the form

$$
\frac{\mathrm{d} P}{\mathrm{~d} r}=-\frac{E+P}{r(r-2 u)}\left(\frac{1}{2} \kappa r^{3} P+u\right) .
$$

To describe the internal state of gas we also use the EoS of the cold Fermi-Dirac neutron gas, which was found by Chandrasekhar [5]. According to this equation, the energy density and pressure equal

$$
\begin{gathered}
E=K(\sinh \tau-\tau), \\
P=\frac{K}{3}\left(\sinh \tau-8 \sinh \frac{\tau}{2}+3 \tau\right),
\end{gathered}
$$

where constant $K=\pi m_{n}^{4} c^{5} /\left(4 h^{3}\right)$ and quantity $\tau$ is related to the Fermi impulse, $p_{F}$, as

$$
\tau=4 \ln \left[\frac{p_{F}}{m_{n} c}+\sqrt{1+\left(\frac{p_{F}}{m_{n} c}\right)^{2}}\right] .
$$

$m_{n}$ is the rest mass of neutron and $h$ is the Planck constant. We also remind the reverse relation,

$$
p_{F}=m_{n} c \sinh \frac{\tau}{4} \text {. }
$$

Using the functions $\tau$, $u$, and $v$, the structure of a cold, spherically symmetric, compact object, consisting exclusively of neutrons, can be described according to the TOV model by the differential equations

$$
\begin{aligned}
& \frac{\mathrm{d} \tau}{\mathrm{d} r}=-\frac{4}{r(r-2 u)} \frac{\sinh \tau-2 \sinh (\tau / 2)}{\cosh \tau-4 \cosh (\tau / 2)+3} \cdot\left\{\frac{1}{6} \kappa K r^{3}[\sinh \tau-8 \sinh (\tau / 2)+3 \tau]+u\right\}, \\
& \frac{\mathrm{d} u}{\mathrm{~d} r}=\frac{1}{2} \kappa K r^{2}(\sinh \tau-\tau), \\
& \frac{\mathrm{d} v}{\mathrm{~d} r}=\frac{2}{r(r-2 u)}\left\{\frac{1}{6} \kappa K r^{3}[\sinh \tau-8 \sinh (\tau / 2)+3 \tau]+u\right\} .
\end{aligned}
$$

For energy density and pressure given by relations (7) and (8), quantity $v$ can also be given analytically as the function of $\tau$. When the derivatives of $P$ and $v$ with respect to $r$ in Equation (4) are replaced by those with respect to $\tau$, the equation can be integrated and we obtain

$$
\mathrm{e}^{v}=\frac{C_{v}}{\cosh ^{2}(\tau / 4)},
$$

where $C_{v}$ is an integration constant.

According to the Birkhoff theorem [10], the metrics of vacuum in a vicinity of an arbitrary, but spherically symmetric distribution of matter is described by the solution of the EFEs, which was found by Schwarzschild [11] and is well-known as the outer Schwarzschild solution (OSS). The Birkhoff theorem must be also valid in the exterior of each spherically symmetric NS. In the physical surface of NS, the metrics found as the solution of Equations (11)-(13), i.e. the interior metrics, must be smoothly tailored to the metrics described by the OSS, i.e. the exterior metrics.

In the following, let us outline the derivation of the OSS from the Equation (12) and Equation (13) written with the help of auxiliary quantity $u$. In the vacuum in a vicinity of NS body, there is everywhere valid that $E=0$ and $P=0$, therefore $\tau=0$. For zero value of $\tau$, Equation (12) yields a constant value of quantity $u$, which we denote by $u_{C}$. Using the definition relation (5) for $u$, we can derive 


$$
\mathrm{e}^{\lambda}=\left(1-\frac{2 u_{C}}{r}\right)^{-1}
$$

For $v$ and $u=u_{C}$, Equation (13) can be simplified to form

$$
\frac{\mathrm{d} v}{\mathrm{~d} r}=\frac{2 u_{C}}{r^{2}\left(1-2 u_{C} / r\right)},
$$

which can be analytically integrated. The integration yields

$$
v=\ln \left(1-\frac{2 u_{C}}{r}\right)+v_{C},
$$

where $v_{C}$ is an integration constant. This relation enables to give component $g_{t t}=\mathrm{e}^{v}$ of metric tensor as

$$
\mathrm{e}^{v}=N_{v}\left(1-\frac{2 u_{C}}{r}\right)
$$

We denoted $\mathrm{e}^{v_{C}}=N_{v}$.

Integration of Equations (11)-(13) for the stellar interior yields the values of quantities $u$ (then $\mathrm{e}^{\lambda}$ ) and $\mathrm{e}^{v}$ on the surfaces of stellar body. Let us denote these values by $u_{\text {out }}\left(u_{\text {in }}\right)$ and $\mathrm{e}^{v_{\text {out }}}\left(\mathrm{e}^{v_{\text {in }}}\right)$ in the outer radius, $R_{\text {out }}$, (inner radius, $R_{\text {in }}$ ) of the star. The demand of the continuity of metrics in the outer (inner) surface implies $u_{C}=u_{\text {out }}$ and $\quad \mathrm{e}^{v_{\text {out }}}=N_{\text {out }}\left(1-2 u_{\text {out }} / R_{\text {out }}\right) \quad$ with $\quad N_{v}=N_{\text {out }} \quad\left(u_{C}=u_{\text {in }}\right.$ and $\mathrm{e}^{v_{\text {in }}}=N_{\text {in }}\left(1-2 u_{\text {in }} / R_{\text {in }}\right) \quad$ with $\left.N_{v}=N_{i n}\right)$. Since we require the flat metrics, i.e. $\mathrm{e}^{\lambda} \rightarrow 1$ and $\mathrm{e}^{v} \rightarrow 1$, in a very large distance from the object (i.e. in the limit $r \rightarrow \infty)$, constant $N_{\text {out }}$ determining the metrics in the outer space must equal unity. This is achieved finding (via an iteration) such an input value of $v$ to the integration of Equation (13) that quantity $\mathrm{e}^{v}$ converges just to $1-2 u_{\text {out }} / R_{\text {out }}$ in $r=R_{\text {out }}$.

Relation (14), which is valid inside the NS body $\left(R_{\text {in }} \leq r \leq R_{\text {out }}\right.$ ), yields $\mathrm{e}^{v_{\text {in }}}=\mathrm{e}^{v_{\text {out }}}=C_{v}$, since $\tau=0$ in $r=R_{\text {in }}$ as well as in $r=R_{\text {out }}$. Therefore, it is valid $N_{\text {in }}\left(1-2 u_{\text {in }} / R_{\text {in }}\right)=1-2 u_{\text {out }} / R_{\text {out }}$. From the latter, constant $N_{\text {in }}$ equals

$$
N_{\text {in }}=\frac{1-2 u_{\text {out }} / R_{\text {out }}}{1-2 u_{\text {in }} / R_{\text {in }}} .
$$

The metrics in the physical surfaces of NS is actually continuous, if not only interior and exterior $g_{r r}$ as well as $g_{t t}$ are correspondingly equal, but their derivatives in respect to the radial distance are equal, too. In terms of mathematics, there must be valid

$$
\begin{aligned}
& \left(\mathrm{e}^{\lambda} \frac{\mathrm{d} \lambda}{\mathrm{d} r}\right)_{r=R_{\text {out }}}=\left[-\frac{2 u_{\text {out }}}{\left(1-2 u_{\text {out }} / r\right)^{2} r^{2}}\right]_{r=R_{\text {out }}}, \\
& \left(\mathrm{e}^{v} \frac{\mathrm{d} v}{\mathrm{~d} r}\right)_{r=R_{\text {out }}}=\left(\frac{2 u_{\text {out }}}{r^{2}}\right)_{r=R_{\text {out }}}
\end{aligned}
$$

in the outer surface and analogous conditions have to also be obeyed in the inner surface.

In a large distance from the NS, the gravitational field is weak and we expect its convergence to that described by the Newtonian physics. It is known that this demand is obeyed when $-u_{\text {out }} / r=\Psi / c^{2}$, where $\Psi$ is the Newtonian gravitational potential. It can be explicitly given by $\Psi=-G M / r$, where $M$ is the gravitational mass of the NS. Using the above introduced relations, the latter can be calculated as

$$
M=\frac{c^{2}}{G} u_{\text {out }} \text {. }
$$

We further remind that the rest mass (sum of the rest masses of all neutrons constituting the object), $M_{o}$, of NS in the traditional theory of these objects is given as (e.g. [12])

$$
M_{o}=4 \pi m_{n} \int_{R_{\text {in }}}^{R_{\text {out }}} \frac{n r^{2}}{\sqrt{1-2 u / r}} \mathrm{~d} r,
$$


where $n=n(r)$ is the number density of neutrons given by relation

$$
n=\frac{8 \pi}{3 h^{3}}\left(m_{n} c\right)^{3} \sinh ^{3} \frac{\tau}{4} .
$$

Because of relations (7) and (8), the maximum energy density and maximum pressure occur in the same distance as the maximum of auxiliary function $\tau$ and maximum of the latter is determined by the condition $\mathrm{d} \tau / \mathrm{d} r=0$. At the same time, the derivative $v^{\prime}$ is zero in this distance. This implies zero net gravity, here, and we will refer, hereinafter, to this critical distance as to $r_{o}$.

Analyzing relation (11), the condition $\mathrm{d} \tau / \mathrm{d} r=0$ is satisfied if $(\kappa K / 6) r_{o}^{3}\left[\sinh \tau_{\max }-8 \sinh \left(\tau_{\max } / 2\right)+3 \tau_{\max }\right]+u_{\max }=0$, where the value of auxiliary quantity $\tau$ in the distance of maximum pressure and energy density, as well the zero net gravity, $r_{o}$, is denoted by $\tau_{\max }$. From the last relation, we can calculate the value of function $u$ in $r_{o}$ as

$$
u_{\max }=-\frac{1}{6} \kappa K r_{o}^{3}\left[\sinh \tau_{\max }-8 \sinh \left(\tau_{\max } / 2\right)+3 \tau_{\max }\right] .
$$

\subsection{Some Constraints in the Traditional Representation}

It appears that the Ni's hollow-sphere model of NS can be constructed after ignoring some constraints, which are demanded, explicitly or implicitly, according to the traditional concept of compact objects. Below, we briefly point out to these constraints.

The demand, that the metrics of vacuum in a vicinity of spherical distribution of matter must be that given by the OSS, yields $g_{t t}=-1 / g_{r r}=1+C_{1} / r$, where $C_{1}$ is an integration constant. Since the vacuum inside a spherically symmetric material shell contains the point $r=0$, in which $g_{t t}$ diverges, constant $C_{1}$ is demanded to equal zero, in this region, to remove the central singularity. The demand of $C_{1}=0$ yields the flat metrics inside the shell. In Sect. 8, we discuss the problem of the central singularity and argue that the gauge of $C_{1}=0$ and, therefore, sweeping out the gravity inside the whole internal region of the shell is not necessary. Thus, the demand of the flat spacetime and zero net gravity inside the spherical shell has the character of postulate, in fact. Hereinafter, we refer to this postulate as "P1".

A consequence of postulate $\mathrm{P} 1$ is that the non-rotating NS must be a full sphere, without any inner physical surface. If there was such a surface and postulate P1 valid, the metrics could not be continuous in the radius of this spherical surface, $R_{i n}$. The NS can exist in the form of full sphere only if the gravity in its interior, from the center to the outer surface, is non-zero and oriented inward. The demand of the gravity always oriented inward is, hereinafter, referred to as postulate "P2". It is a further constraint put on the GR when modeling the NS.

Postulate P2 has never be explicitly stated in the literature. It was implicitly established by Oppenheimer and Volkoff [1], when they started the numerical integration to construct the first NS models from the center of the object. Such the processing is possible only if the mass of the star is distributed down to the center and this is possible only if the gravity is oriented inward in the entire stellar interior.

The Ni's solution of TOV equations is possible if the postulates P1 and P2 are abolished. In the following sections, we try, beside other, to provide a reason for their abolition within the GR.

\section{An Example of Neutron-Star Model Constructed by Using the Ni's Solution}

In this section, let us introduce the basic properties of the NS model constructed by using the Ni's solution of Equations (11)-(13) in more detail. We numerically integrate these equations starting, for example, in the star-centric distance $10 \mathrm{~km}$. We identify this distance to the distance $r_{o}$ of zero net gravity, therefore the input value of auxiliary quantity $u$ is identical to $u_{\max }$, which is given by Equation (25). Let us consider the input value of the Fermi impulse equal to, e.g., $1.2 m_{n} c$. It implies the corresponding input value $\tau_{\max }$ according to relation (9). The integration is performed in two parts: in inward direction and outward direction. We arbitrarily terminate each part when $\tau$ decreases below $10^{-4}$ to avoid a transgress to a negative value of $\tau$ in the last integration step.

The input value of auxiliary function $v$ is found via iteration to be equal to -3.488263 . For this input value of $v$, the outward processed integration of Equation (13) ends just with value $v_{\text {out }}=1-2 u_{\text {out }} / R_{\text {out }}$ and, thus, the metrics is continuous in the outer physical surface of NS. 
The behaviors of density (calculated as the ratio of $E / c^{2}$ ) and components $g_{r r}$ and $g_{t t}$ of metric tensor, as well as auxiliary quantity $u$ in this example are shown in Figure 1. From the maximum in $r_{o}$, the density (Figure 1(a)) decreases in as inward as outward processed integration. (The behavior of pressure is qualitatively the same.) In the interval of distances $R_{\text {in }}<r<r_{o} \quad\left(r_{o}<r<R_{\text {out }}\right)$, the gradient of the density is positive (negative). On contrary, the derivative of function $g_{t t}$ (Figure 1(c)) with respect to $r$, which determines the orientation of the gravitational action (see the gravitational acceleration given by relation (40); it is proportional to $v^{\prime}$ ), is negative (positive) in the same interval.

The metrics in the external vacuum is described by relations (15) and (18) with $u_{o}=u_{\text {out }}$ and $N_{v}=N_{\text {out }}=1$ in the outer space. Similarly, $u_{o}=u_{i n}$ and $N_{v}=N_{\text {in }}$ given by relation (19) in the inner space. Specifically,

$$
\begin{aligned}
& g_{r r}\left(r \geq R_{\text {out }}\right)=-\frac{1}{1-2 u_{\text {out }} / r}=-\frac{1}{1-2\left|u_{\text {out }}\right| / r}, \\
& g_{r r}\left(r \leq R_{\text {in }}\right)=-\frac{1}{1-2 u_{\text {in }} / r}=-\frac{1}{1+2\left|u_{\text {in }}\right| / r}, \\
& g_{\text {tt }}\left(r \geq R_{\text {out }}\right)=1-\frac{2 u_{\text {out }}}{r}=1-\frac{2\left|u_{\text {out }}\right|}{r}, \\
& g_{\text {tt }}\left(r \leq R_{\text {in }}\right)=N_{\text {in }}\left(1-\frac{2 u_{\text {in }}}{r}\right)=N_{\text {in }}\left(1+\frac{2\left|u_{\text {in }}\right|}{r}\right) .
\end{aligned}
$$
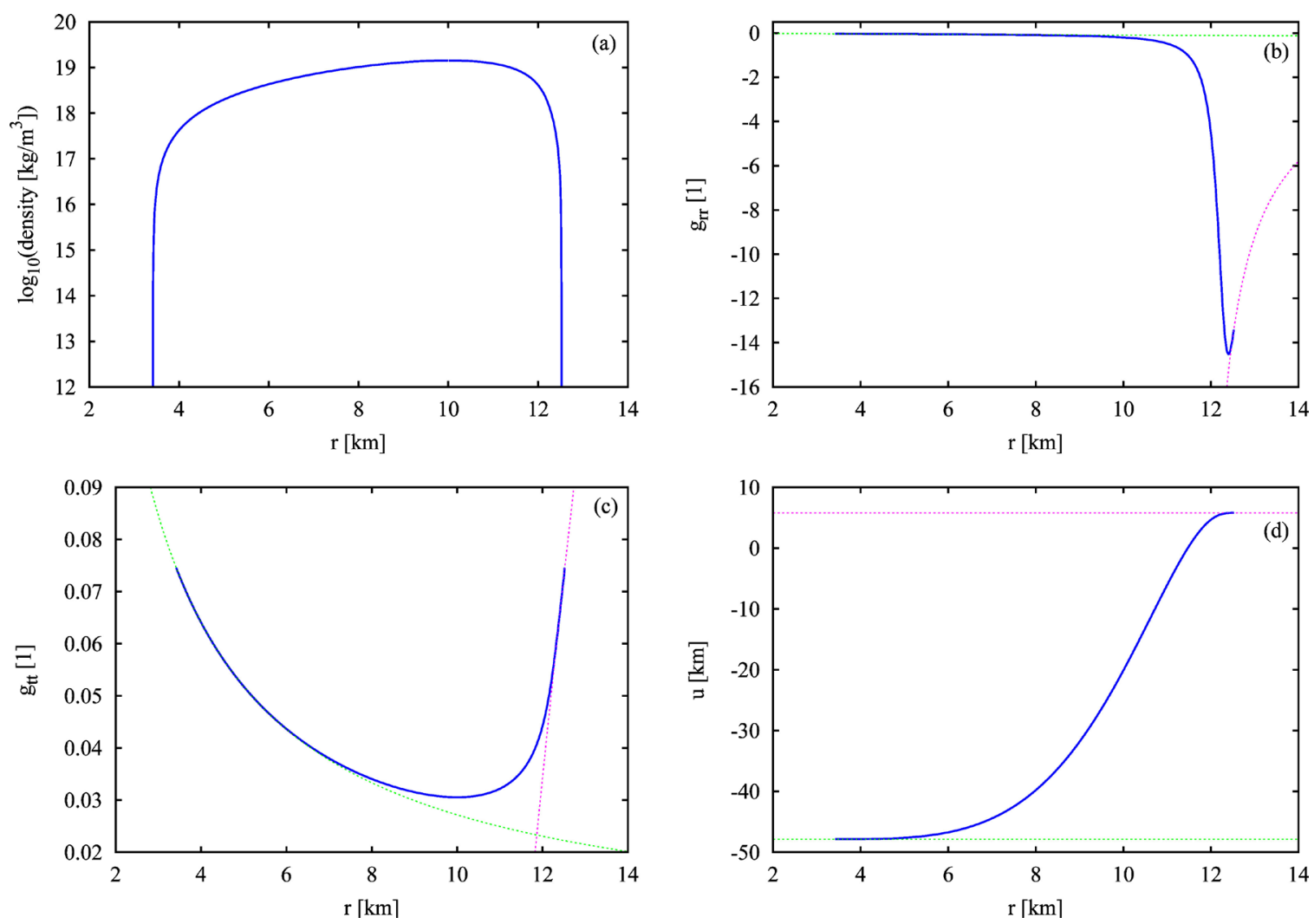

Figure 1. The behavior of the density (plot a), components $g_{r r}$ (b) and $g_{t t}$ (c) of metric tensor, and auxiliary quantity $u$ (d) in the example of compact, pure-neutron object discussed in Section 3. The thick solid blue curve shows the behavior inside the compact-object body in all plots. In plots (b)-(d), the dotted green curve, touching the solid blue curve in its left end-point, shows the outer Schwarzschild solution tailored to the former behavior in the inner radius and dotted violet curve, touching the solid blue curve in its right end-point, shows the tailoring of the metrics in the outer physical radius of the object. The mass of the neutron object in this example is $3.923 \mathrm{M}_{\odot}$. 
In Figure 1, one can see that this metrics is perfectly tailored with that valid for the NS's interior. It means that the metrics is perfectly continuous in both outer and inner physical surfaces. Not only the values of $g_{r r}$, $g_{t t}$, and $u$ are tailored, but their derivatives also equal each other correspondingly. Ni [3] assumed the Minkowski metrics in the internal void, $r \leq R_{\text {in }}$, which was not however well tailored with the NS-body metrics.

The negative derivative of $g_{t t}$ with respect to $r$ in the interval $r<r_{o}$ (also in $r<R_{\text {in }}$ ) implies an outward oriented net gravity, there. Such the behavior is reasoned in the following sections.

The stable configuration of matter in the interval from $R_{i n}$ to $r_{o}$ occurs due to the change of the orientation of gas pressure gradient, which balances the outward acting gravity. The fact that the orientation of this gradient is always opposite to gravity can be seen in relation (4). Since there is always valid $E+P \geq 0$, the value of gradient $\mathrm{d} P / \mathrm{d} r$ has always the opposite sign than the value of $v^{\prime}$ and, therefore, the gradient is opposite. We remind that the gravity is proportional to $v^{\prime}$ and its orientation to the sign of this derivative.

\section{Spherical Symmetry and Gravity of Outer Layers}

In course to explain the outward acting gravitational attraction, let us, firstly, to deal with an important consequence of spherical symmetry in the Newtonian physics. In this theory, the space is Euclidean regardless the amount, compactness, and radial distribution of matter in the spherically symmetric object. Every such the object can then be divided into a large number of thin concentric material layers. The net gravitational force from every layer can be calculated separately and the total net force is simply the sum of the partial forces from every layer.

Let us consider an infinitesimally small material element of a thin, spherically symmetric layer in a distance $r_{1}$ from a test particle, which is situated inside the layer. In this distance, let the element is seen within a certain small space angle. Its volume is then proportional to $r_{1}^{2}$. According to the Newton force law, the gravitational force between the element and the test particle is proportional to $r_{1}^{-2}$, therefore the product of $r_{1}^{2}$ and $r_{1}^{-2}$ occurs in the formula for this force. The product equals unity, i.e. it is constant. The analogous force can be found between the test particle and small material element situated in exactly opposite direction and seen within the same space angle as the first element. If the second element is in distance $r_{2}$ from the test particle, its volume is proportional to $r_{2}^{-2}$ and the corresponding force is proportional to the product of $r_{2}^{2}$ and $-r_{2}^{-2}$ (sign minus expresses the opposite orientation of force). This product equals -1 , i.e. the same constant, but with the opposite sign. Therefore, if we add both partial forces, their sum is exactly zero.

In other words, the force from the element in the given direction is eliminated by the force from the element in the opposite direction. For every element, it is possible to find the element in the opposite direction, exactly eliminating the force of the first element, therefore the total net gravity of the thin spherical layer on the test particle wherever inside it is exactly zero. Consequently, there can be whatever number of outer concentric layers, with the radii larger than the object-centric distance of given test particle, their net gravitational force is the sum of zeros and, hence, zero.

However, it is important to realize that this calculation, with zero result, can be made only in the Euclidean space of Newtonian physics. Only in this case, it can be exactly and generally proved (analytically calculated) that the net gravity of the outer layers of spherically symmetric object is zero.

If we consider a curved spacetime or curved space in a static problem in the GR, there occur two differences, which yield problems. At first, the GR is not linear, therefore we cannot divide any spherical object into a number of thin concentric layers, calculate their partial gravitational actions separately, and obtain the net action by adding the partial results. At second, the distribution of matter, which is seen as spherically symmetric by the observer in its center, is not longer seen, in general, as spherically symmetric by an observer aside the center. The curved space changes the distance to a material element when seen by the observers in different places, in general.

In a calculation of gravitational acceleration of a test particle inside a spherical layer, the element volume is not longer proportional to the quadrate of distance in the curved space. And, the gravitational acceleration cannot be expected to be reciprocally proportional to this quadrate, either. Unless the gravity of outer layers is postulated to be zero, it cannot be expected to be zero, except of some special cases, perhaps. The Ni's solution implies that this gravity is actually non-zero and oriented outward from the center of the object, which is seen as spherically symmetric by the observer in its center.

\section{Analysis of Gravitational Potential}

The EFEs are solved via integrations, which produce the integration constants. In an application of the solution 
of EFEs to a real object, we need to physically represent these constants and find their values. In this process, the fact that the GR must converge to its Newtonian approximation in the limit of weak field was utilized.

Sometimes, it seems that the representations of both these theories are not distinguished, properly. The rules being valid within the classical Newtonian physics are used in an argumentation within GR. Consequently, the GR is, in fact, constrained by these rules if one requires their validity in both theories. Below, we discuss this problem in more detail in the context of the Ni's solution and demonstrate the consequences in the case when the GR is completely deliberated from the Newtonian physics, in this case. It appears that the most important quantity in this discussion is the gravitational potential, its understanding and definition.

\subsection{Potential in Newtonian Physics}

When we speak about the gauging of the constants produced by the integrations of the EFEs, the most common example is, likely, the gauging of the constants in the OSS, which describes the vacuum metrics shaped by a point-like particle. This metrics is identical to the metrics of vacuum above the outer surface of the spherically symmetric object. From the integration of the corresponding EFEs, we obtained the components of metric tensor $g_{r r}=-\mathrm{e}^{\lambda}$ and $g_{t t}=\mathrm{e}^{v}$ given by relations (15) and (18) in Section 2.1, where $u_{C}$ and $N_{v}$ were the integration constants. In the region of radial distance $r>R_{\text {out }}$, the integration constants were denoted by $u_{C}=u_{\text {out }}$ and $N_{v}=N_{\text {out }}$. In the limit $r \rightarrow \infty$, the metrics is demanded, as mentioned in Section 2.1, to converge to the flat metrics, i.e. $g_{r r} \rightarrow-1$ and $g_{t t} \rightarrow 1$. Hence, constant $N_{\text {out }}=1$.

In the limit of weak field, the gravitational acceleration of a test particle is oriented toward the center of the acting object and, therefore, toward the origin of the coordinate frame considered. In this limit, it is calculated as (e.g., [13])

$$
\ddot{\boldsymbol{r}} \approx-\frac{c^{2}}{2} \frac{\partial g_{\text {tt }}}{\partial r} \approx-\frac{c^{2}}{2} \frac{\partial}{\partial r}\left(1-\frac{2 u_{\text {out }}}{r}\right) \approx \frac{\partial}{\partial r}\left(\frac{c^{2} u_{\text {out }}}{r}\right) .
$$

Sign minus respects the fact that gravity is attractive force and the acceleration is oriented against the direction of coordinate $r$-axis. The analogous formula in the Newtonian physics is

$$
\ddot{r}=-\operatorname{grad} \Psi=-\frac{\partial \Psi}{\partial r},
$$

in the considered radial case. $\Psi$ is the gravitational potential. Comparing the last relations, we can gauge constant $u_{\text {out }}$ as

$$
\frac{u_{\text {out }}}{r}=-\frac{\Psi}{c^{2}} .
$$

This is the "classical" gauging, which implies that there must always be valid $u_{\text {out }}>0$, because the gravitational potential $\Psi<0$ and, hence, $-\Psi / c^{2}>0$.

We know that $\Psi=-G M / r$ for the object of mass $M$, therefore $u_{\text {out }}=G M / c^{2}$ as already found in Section 2.1. If the formulas are expressed in the system of units with the unit gravitational constant, $G$, and unit speed of light, $c$, then one can obtain an apparent identity $u_{\text {out }}=M$. In such the case, it is however necessary remember that fraction $2 M / r$, which then occurs in the GR formulas, is not double of mass over radial distance, but the gravitational constant and quadrate of the light speed are also implicitly present. (With respect to the apparent identity $u_{\text {out }}=M$, it used to be sometimes reasoned that $u_{\text {out }}$ must be positive, since the mass, $M$, must always be the positive quantity.)

In the classical Newtonian physics, the gravitational potential used to be defined as the negative of the integral of corresponding gravitational acceleration from a given radial distance $r$ to infinity. In the inverse calculation, the acceleration is calculated as the negative of the gradient of potential. Because the reference point in infinity is the matter of convention, some authors prefer to consider rather a change of the potential when a test particle is moved from a point of space to other point. So, they define the change of potential, which is related to the corresponding change in the potential energy of the particle. This definition is unique, no reference point is necessary.

For example, if the test particle is ejected radially outward from the surface of spherically symmetric object, having its upper radius $R_{\text {out }}$, to a distance $r$, the difference of the potential energy is 
$W_{p}(r)-W_{p}\left(R_{\text {out }}\right)=m_{1} \Psi(r)-m_{1} \Psi\left(R_{\text {out }}\right)$, where $m_{1}$ is the mass of the particle. If we supply $\Psi(r)=-G M / r$, the difference of the potential energy can be given as $G m_{1} M\left(1 / R_{\text {out }}-1 / r\right)$. Since $r>R_{\text {out }}$, the difference is positive as necessary, because we know that the speed of the outward ejected particle has to decrease, therefore its kinetic energy decreases and, consequently, the potential energy has to increase to keep the total energy constant according to the energy conservation law.

\subsection{Two Concepts of Potential in General Relativity}

To describe the motion of a test particle moving in the vacuum in the static spacetime shaped by a stable concentration of matter, the so-called "effective potential", $\tilde{U}$ is used. Let us denote the quadrate of the particle's speed by $(\mathrm{d} r / \mathrm{d} T)^{2}$ and its energy per unit mass as $\tilde{E}$ ( $T$ is the proper time). The effective potential is related to these quantities as [12]

$$
\left(\frac{\mathrm{d} r}{\mathrm{~d} T}\right)^{2}+\tilde{U}^{2}=\tilde{E}^{2}
$$

In the metrics described by the OSS, the effective potential is given as $\tilde{U}=\left[\left(1-2 u_{C} / r\right)\left(1+L / r^{2}\right)\right]^{1 / 2}$, where $u_{C}$ is constant and $L$ is the angular momentum. In our radial case, $L=0$, therefore the effective potential is

$$
\tilde{U}=c^{2} \sqrt{1-\frac{2 u_{C}}{r}} .
$$

On the other-hand side, we used, in the previous section, the classical Newtonian potential to gauge the auxiliary metric and, therefore, GR quantity $u$. Thus, this is the second potential sometimes figuring in the GR as the ordinary quantity.

There is no problem, if this is done only in the weak field (and $r>R_{\text {out }}$ ) and only the numerical value of $u$, without the physical representation, is found. However, if the representation of $u$ as the quantity of closely related to the potential is made and it is argued that $u$ must behave accordingly to this potential wherever inside the NS, not only in a weak field far from it, the second potential becomes the integral part of the GR. But, should the GR really be constrained by the demand to satisfy all (from Newtonian physics originating) properties of this quantity?

Notice that while there is demanded $u>0$ because the Newtonian potential has to be positive, the effective potential is positive also for $u=u_{C}<0$. And, the effective potential can be written with the help of the Newtonian potential, $\Psi$, as $U=c^{2} \sqrt{1+2 \Psi / c^{2}}$. Is this dependence of "potential on potential" normal and really needed in the GR?

The effective potential was established for the vacuum outside the material object. To generalize the effective potential for whole space, i.e. from $r=0$ to infinity, in the case of the Ni's model of NS, we establish a new gauging of the constant term of this quantity. So, let us now to repeat the well-known derivation of the GR effective potential using the definition that the potential is the integral of corresponding gravitational acceleration. Thus, the first task is the determination of the acceleration. We again consider the spherical coordinate system, $r$, $\vartheta$, and $\varphi$. In the case of spherical symmetry, the line element can be given as

$$
\mathrm{d} s^{2}=g_{i i}\left(\mathrm{~d} x^{i}\right)^{2}=-\mathrm{e}^{\lambda} \mathrm{d} r^{2}-r^{2} \mathrm{~d} \vartheta^{2}-r^{2} \sin ^{2} \vartheta \mathrm{d} \varphi^{2}+c^{2} \mathrm{e}^{v} \mathrm{~d} t^{2} .
$$

The four-vector of acceleration is related to the metrics as

$$
\frac{\mathrm{d}^{2} x^{i}}{\mathrm{~d} s^{2}}+\Gamma_{j k}^{i} \frac{\mathrm{d} x^{j}}{\mathrm{~d} s} \frac{\mathrm{d} x^{k}}{\mathrm{~d} s}=0,
$$

where $\Gamma_{j k}^{i}$ are the Christoffel symbols and indices $i, j$, and $k$ range from 1 to 4 . Here, we also use the alternative denotation, with the numerical indices, whereby $g_{11}=g_{r r}, g_{44}=g_{t t}, x^{1}=r, x^{2}=\vartheta, x^{3}=\varphi$, and $x^{4}=c t$.

Further, we consider exclusively the radial acceleration of a static test particle. It means, $\mathrm{d} r / \mathrm{d} t=\mathrm{d} \vartheta / \mathrm{d} t=\mathrm{d} \varphi / \mathrm{d} t=0$. In this case, only the single component of four-velocity, figuring in relation (36), is 
non-zero. Specifically,

$$
c \frac{\mathrm{d} t}{\mathrm{~d} s}=\mathrm{e}^{-v} .
$$

Hence, relation (36) can be simplified and four-acceleration can be given as

$$
\frac{\mathrm{d}^{2} x^{i}}{\mathrm{~d} s^{2}}=-\Gamma_{44}^{i} c^{2}\left(\frac{\mathrm{d} t}{\mathrm{~d} s}\right)^{2}
$$

Of the Christoffel symbols $\Gamma_{44}^{1}, \Gamma_{44}^{2}, \Gamma_{44}^{3}$, and $\Gamma_{44}^{4}$, figuring in the last relation, only the first symbol is non-zero, whereby [13]

$$
\Gamma_{44}^{1}=\frac{1}{2} \mathrm{e}^{v-\lambda} v^{\prime}
$$

Therefore, only the component $\mathrm{d}^{2} x^{1} / \mathrm{ds}^{2}=\mathrm{d}^{2} r / \mathrm{ds} s^{2}$ of the four-acceleration does not equal to zero. Using relation (38) and replacing $\mathrm{ds}^{2}$ by $\mathrm{ds}^{2}=c^{2} \mathrm{~d} T^{2}$, where $\mathrm{d} T$ is the element of proper time, we gain the acceleration in the radial direction we wanted to derive:

$$
\frac{\mathrm{d}^{2} r}{\mathrm{~d} T^{2}}=-\frac{c^{2}}{2} \mathrm{e}^{-\lambda} v^{\prime}
$$

The corresponding gravitational potential is integral of this quantity. In the integration, we have to remember the GR contraction of length and, thus, to integrate through the proper element of length $\mathrm{e}^{\lambda / 2} \mathrm{~d} r$ (instead of the Schwarzschild-coordinate element $\mathrm{d} r$ ).

\subsection{New GR Gravitational Potential}

The potential energy is the negative of the work done against the gravity. In a full-sphere object with the gravitational action everywhere oriented inward, the force to do the work has to be oriented outward. When we calculate the change of the potential energy, the integration is made from a point nearer to the full-sphere object center to a point, which is in a larger distance.

In a hollow-sphere object, the above mentioned direction of the integration to calculate the potential energy and corresponding potential is relevant only in the interval of radial distance from $r_{o}$ to infinity. Because the gravitational action is opposite in the complementary interval, from 0 to $r_{o}$, we have to make the integration in this, opposite direction, i.e. from a more distant to a less distant point from the center, in this region.

If one wishes to give a reference point for the calculation of potential generated by hollow-sphere object, the most appropriate is a point just in distance $r_{o}$. This distance appears to be a natural starting point of each integration to calculate the potential, regardless the end point is in distance $r>r_{o}$ or in $r<r_{o}$. Using this definition, we propose the new form of the effective potential in the GR, which can mathematically be expressed as

$$
U(r)=-\int_{r_{o}}^{r} \frac{\mathrm{d}^{2} r}{\mathrm{~d} T^{2}} \mathrm{e}^{\lambda / 2} \mathrm{~d} r=\frac{c^{2}}{2} \int_{r_{o}}^{r} \mathrm{e}^{-\lambda / 2} v^{\prime} \mathrm{d} r .
$$

If $r<R_{i n}$, it is useful to divide integral (41) into two integrals. The first integration runs from $r_{o}$ to $R_{i n}$ and the second from $R_{i n}$ to $r$. Let us denote the result of the first integral by $U_{d}$. This integration has to be performed numerically. In the second integral, components of metric tensor $\mathrm{e}^{\lambda}$ and $\mathrm{e}^{v}$ are given by the OSS and $u=u_{i n}$ is constant. So, we can re-write relation (41) to

$$
U\left(r<R_{i n}\right)=U_{d}+\frac{c^{2}}{2} \int_{R_{i n}}^{r} \frac{1}{\sqrt{1-2 u_{i n} / r}} \mathrm{~d} r=U_{d}-c^{2} \sqrt{1-\frac{2 u_{i n}}{R_{i n}}}+c^{2} \sqrt{1-\frac{2 u_{i n}}{r}} .
$$

If $r>R_{\text {out }}$, we can analogously divide integral (41) into two parts. This time, the first integration runs from $r_{o}$ to $R_{\text {out }}$ and the second from $R_{\text {out }}$ to $r$. Let us denote the result of the first, again numerical integration by $U_{u}$ and use the OSS to express the components $\mathrm{e}^{\lambda}$ and $\mathrm{e}^{v}$ of metric tensor in the second integral. Here, 
$u=u_{\text {out }}$ is again constant. The form of relation (41) changes to

$$
U\left(r>R_{\text {out }}\right)=U_{u}+\frac{c^{2}}{2} \int_{R_{\text {out }}}^{r} \frac{1}{\sqrt{1-2 u_{\text {out }} / r}} \mathrm{~d} r=U_{u}-c^{2} \sqrt{1-\frac{2 u_{\text {out }}}{R_{\text {out }}}}+c^{2} \sqrt{1-\frac{2 u_{\text {out }}}{r}} .
$$

In the last formula, term $U_{u}$ can be regarded as constant (it does not change, when we investigate a change of potential energy of a test particle in region $r>R_{\text {out }}$ ) and we can see that the dependence of the potential on radial distance, $r$, is the same as in the effective potential introduced in the beginning of Section 5.2, i.e. $U \propto c^{2} \sqrt{1-2 u_{\text {out }} / r}$. The new potential, corresponding to the effective potential in region $r>R_{\text {out }}$, differs from the later by its constant part $U_{u}-c^{2} \sqrt{1-2 u_{\text {out }} / R_{\text {out }}}$. Such a difference is not important from the physical point of view, because it disappears, when we calculate a difference of potential in two points. We know that there is also a difference in the constant term between the Newtonian potential, $\Psi=-G M / r$, and approximation of the effective potential for a weak field, which equals $\tilde{U} \approx c^{2}-G M / r$ (assuming the gauging of $u_{C}$ in relation (34) as $\left.u_{C}=G M / c^{2}\right)$. The approximation of the effective potential contains constant term $c^{2}$, which absents in its Newtonian counterpart. Anyway, both forms can be used in a practical calculation of energy gain or loss, because they give the same result.

The behavior of the new potential in the example of Ni's hollow sphere presented is Section 3 is shown in Figure 2. We can observe that it acquires only positive values in the entire interval of radial distance (and zero in $r=r_{o}$ ).

To establish the new potential, which is mathematically expressed by Formula (41), we propose to rule out the physical quantity "mass" from the GR and regard the quantity $u$, established originally by Oppenheimer and Volkoff [1], as only an auxiliary metric quantity. In accord with its original definition (see relation (5)), this quantity replaces the auxiliary metric quantity $\lambda$ in the EFEs and, therefore, it can be represented as the alternative parameter characterizing the metrics. In this new representation, the conversion of $u_{\text {out }}$ to mass $M$ according to relation (22) is only formal, giving one aspect of metrics. We suggest to strictly distinguish between mass $M$, which is the quantity existing within the Newtonian physics, and GR parameter $u$ (also $u_{\text {out }}$ ). Although both parameters interact in the gauging, its physical meaning is different and should not be treated as identical (when unit $G$ and $c$ are used).

If we regard the negative values of $u$ as physically acceptable, we accept, in fact, that the size of $g_{r r}$-component of metric tensor, $\left|g_{r r}\right|=\mathrm{e}^{\lambda}$, can also be smaller than unity. Actually, there is no reason in the GR of why the inequality $\mathrm{e}^{\lambda}>1$ should always be valid.

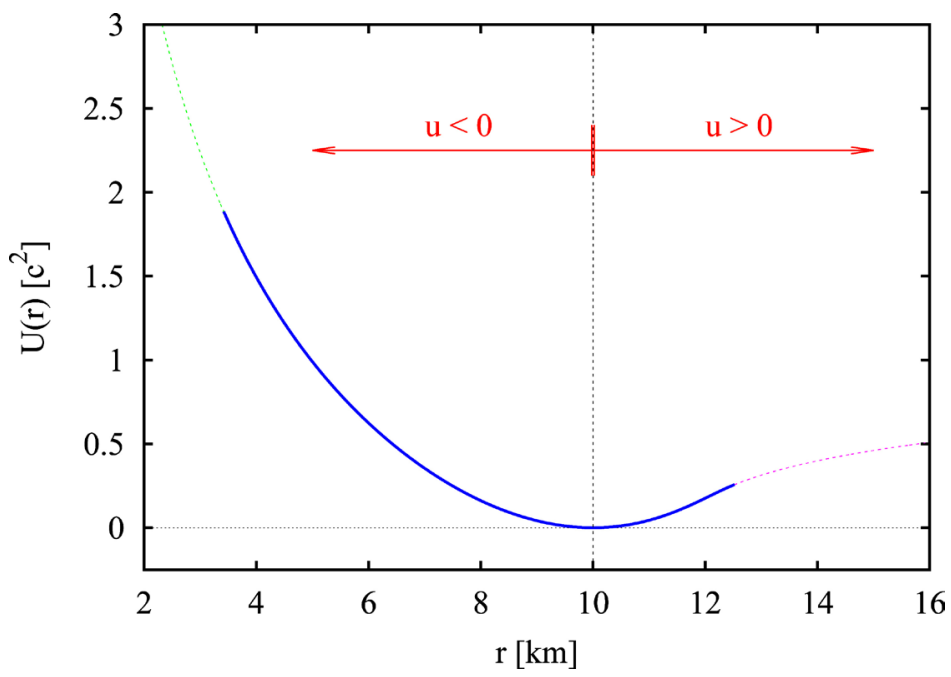

Figure 2. The behavior of the newly defined gravitational potential in the GR in the example of the Ni's hollow sphere presented in Section 3. The blue thick solid curve shows the potential inside the object's body. This potential is calculated within the numerical integration of the EFEs by using relation (41). The dashed green (dashed violet) curves shows the potential in the region of radial distance $r<R_{\text {in }} \quad\left(r>R_{\text {out }}\right)$, which is calculated by relation (42) (relation (43)). 
Within the GR, the concepts of energy (of all kinds) and potential remain. Since it has been empirically found that constant $u_{i n}$, appearing in the solutions of EFEs exclusively in the region $r \leq R_{i n}$, is always negative, arguments of the second square roots in relation (42) are always positive, therefore the potential is always real-valued.

When the quantity $u$ is regarded as the auxiliary quantity characterizing the metrics, the new gravitational potential can be proved as always positive quantity, even in the region where $u<0$. Thus, the requirement of the physically acceptable gravitational potential is obeyed in the whole space, from the center of hollow sphere up to an infinite distance.

\subsection{Remarks Concerning the Mass Elimination}

To support our suggestion from the previous subsection about the ruling out the mass as the physical quantity from the GR, we remind the suggestion, published in our earlier paper (arXiv: 1206.0405v1 [physics.gen-ph]), to eliminate not only the mass from the physics, but the electric charge and some fundamental physical constants as well.

We know, the fundamental laws, like the Newton force law, Coulomb law, or law of inertia, can exclusively be experimentally verified in a combination of one, e.g. Newton, force law and the law of inertia. In other words, we can verify the prediction made by the solution of the corresponding equation of motion. So, the real physical theory is never represented by a single law, but by an equation (or a set of equations).

Let us explain the principle of the application of fundamental physical laws in the example of two electrically charged, static particles. In this example, the laws are used to describe their dynamics. We determine the acceleration of the first, "test" particle (TP), when influenced by the second, "acting" particle (AP). The TP has mass $m_{T}$ and charge $q_{T}$ and AP has mass $m_{A}$ and charge $q_{A}$. The AP acts on the TP by its gravity as well as electrically. Initially, both particles are assumed to be in rest in mutual distance $r$. The relevant equation of motion for the TP reads

$$
m_{T} \frac{\Delta v}{\Delta t}=-\frac{G m_{T} m_{A}}{r^{2}}+\frac{1}{4 \pi \varepsilon_{o}} \frac{q_{T} q_{A}}{r^{2}},
$$

where $\Delta v$ is the change of the velocity of the TP due to the action of the AP during a short time interval $\Delta t$ and $\varepsilon_{0}$ is the permittivity of vacuum (the equation is given in the SI units).

We assume that the AP and TP are composed of matter consisting only of elementary particles of $k$ kinds, whereby the mass of $j$-th kind is $m_{j}$. While the AP consists of $N_{j}$ particles of $j$-th kind, the TP consists of $n_{j}$ particles of $j$-th kind. Taking into account such the composition, the masses $m_{A}$ and $m_{T}$ can be given as $m_{A}=\sum_{j=1}^{k} N_{j} m_{j}$ and $m_{T}=\sum_{j=1}^{k} n_{j} m_{j}$.

Further, if the AP consists of $N_{+}\left(N_{-}\right)$carriers of positive (negative) elementary charge and the TP consists of $n_{+}\left(n_{-}\right)$carriers of positive (negative) elementary charge, then the charges $q_{A}$ and $q_{T}$ can be given as $q_{A}=\left(N_{+}-N_{-}\right) q_{o}$ and $q_{T}=\left(n_{+}-n_{-}\right) q_{o}$. The elementary electric charge (charge of proton) is denoted by $q_{o}$. Now, Equation (44) can be re-written to

$$
\left(\sum_{j=1}^{k} n_{j} m_{j}\right) \frac{\Delta v}{\Delta t}=-\frac{G}{r^{2}}\left(\sum_{j=1}^{k} n_{j} m_{j}\right)\left(\sum_{j=1}^{k} N_{j} m_{j}\right)+\frac{q_{o}^{2}}{4 \pi \varepsilon_{o} r^{2}}\left(n_{+}-n_{-}\right)\left(N_{+}-N_{-}\right) .
$$

The dimensional analysis enables combining the fundamental physical constants to obtain special length, time, and mass, which are known as the Planck length, $L_{P}$, Planck time, $t_{P}$, and Planck mass, $M_{P}$. Specifically, these quantities are defined by

$$
L_{P}=\sqrt{\frac{G \hbar}{c^{3}}}, \quad t_{P}=\sqrt{\frac{G \hbar}{c^{5}}}, \quad M_{P}=\sqrt{\frac{\hbar c}{G}},
$$

where $\hbar$ is the Planck constant, $h$, divided by $2 \pi$. The Planck time is supposed to be the shortest time interval in the universe. So, there should not be problem to identify the time interval $\Delta t$ in Equation (44) to the Planck time, i.e. to put $\Delta t=t_{p}$.

In quantum physics, a wave is associated to every elementary particle. The angular frequency, $\omega$, of this wave is related to the particle's mass, $m$, according to the de Broglie's relation $\hbar \omega=m c^{2}$ [14] [15]. Since the 
angular frequency is related to the wavelength, $b$, as $\omega=2 \pi c / b$, mass $m$ can also be given as

$$
m=\frac{2 \pi \hbar}{c b} \text {. }
$$

If we use this relation to convert masses $m_{j}$ to the corresponding wavelengths $b_{j}$, and assume the identity $\Delta t=t_{p}$, equation of motion (45) changes to

$$
\left(\sum_{j=1}^{k} n_{j} \frac{2 \pi \hbar}{c b_{j}}\right) \sqrt{\frac{c^{5}}{G \hbar}} \Delta v=-\frac{G}{r^{2}}\left(\sum_{j=1}^{k} n_{j} \frac{2 \pi \hbar}{c b_{j}}\right)\left(\sum_{j=1}^{k} N_{j} \frac{2 \pi \hbar}{c b_{j}}\right)+\frac{q_{o}^{2}}{4 \pi \varepsilon_{o} r^{2}}\left(n_{+}-n_{-}\right)\left(N_{+}-N_{-}\right) .
$$

In the last term, the fraction $q_{o}^{2} /\left(4 \pi \varepsilon_{o} r^{2}\right)$ can be re-written with the help of the dimensionless fine-structure constant, $\alpha=q_{o}^{2} /\left(4 \pi \varepsilon_{o} \hbar c\right)$, as $\alpha \hbar c / r^{2}$. Taking this possibility into account, using the relation giving the Planck length, and multiplying (48) by $L_{P}^{2} /(2 \pi \hbar c)$, this equation can be written in the form

$$
\left(\sum_{j=1}^{k} n_{j} \frac{L_{P}}{b_{j}}\right) \frac{\Delta v}{c}=-2 \pi\left(\frac{L_{P}}{r}\right)^{2}\left(\sum_{j=1}^{k} n_{j} \frac{L_{P}}{b_{j}}\right)\left(\sum_{j=1}^{k} N_{p} \frac{L_{P}}{b_{j}}\right)+\frac{\alpha}{2 \pi}\left(\frac{L_{P}}{r}\right)^{2}\left(n_{+}-n_{-}\right)\left(N_{+}-N_{-}\right) .
$$

We can see that the last equation is dimensionless and the fundamental constants $G, \varepsilon_{o}$, and $\hbar$ disappeared. Only the dimensionless fine-structure constant and the ratios of various lengths and velocities figures there, except of the numbers of particles and charge carriers. Because the universe is not static, the occurrence of the velocities, which represent the change (the first derivative of length) and change of change (the second derivative of length), is obvious in the description.

The fundamental equations yielding the TOV model (11)-(13) can also be re-written to the dimensionless form. If we denote the wavelength of the wave associated with neutron by $b_{n}$, then the product of constants $\kappa=8 \pi G / c^{4}$ and $K=\pi m_{n}^{4} c^{5} /(4 h)$, which equals $\kappa K=2 \pi^{2} G m_{n}^{4} c / h^{3}$, can be converted, using Formula (47), to $\kappa K=4 \pi^{3} L_{P}^{2} / b_{n}^{4}$. With this new form of $\kappa K$ and after multiplication of Equations (11) and (13) by $L_{P}$, the three fundamental equations acquire form

$$
\begin{aligned}
& L_{P} \frac{\mathrm{d} \tau}{\mathrm{d} r}=-\frac{2}{1-2 u / r} \frac{L_{P}}{r} \frac{\sinh \tau-2 \sinh (\tau / 2)}{\cosh \tau-4 \cosh (\tau / 2)+3} \\
& \cdot\left\{\frac{4 \pi^{3}}{3}\left(\frac{L_{P}}{b_{n}}\right)^{4}\left(\frac{r}{L_{P}}\right)^{2}[\sinh \tau-8 \sinh (\tau / 2)+3 \tau]+\frac{2 u}{r}\right\}, \\
& \frac{\mathrm{d} u}{\mathrm{~d} r}=2 \pi^{3}\left(\frac{L_{P}}{b_{n}}\right)^{4}\left(\frac{r}{L_{P}}\right)^{2}(\sinh \tau-\tau), \\
& L_{P} \frac{\mathrm{d} v}{\mathrm{~d} r}=\frac{1}{1-2 u / r} \frac{L_{P}}{r}\left\{\frac{4 \pi^{3}}{3}\left(\frac{L_{P}}{b_{n}}\right)^{4}\left(\frac{r}{L_{P}}\right)^{2}[\sinh \tau-8 \sinh (\tau / 2)+3 \tau]+\frac{2 u}{r}\right\} .
\end{aligned}
$$

Because this is the static problem, the equations contain only the ratios of lengths and derivatives with respect to length.

The GR was originally intended to be formulated as a geometric theory and Equation (49) as well as Equations (50)-(52) indicate the way toward the GR as the pure geometric theory. Mass does not seem to be any component of such a theory. Hence, our proposal to rule out this quantity from any GR description and argumentation appears to be reasonable.

We note that such the units as meter, second, or kilogram were defined by man and, thus, cannot be regarded as the "natural" physical units. As well, the quantities as mass or electric charge, which were also established by man, can be, in fact, only the artificial quantities not really existing in the nature. Instead, the quantities as length, frequency, their change, and change of their change seem to be natural. And, concerning the physical units, the above mentioned Planck length, Planck time, and speed of light are, likely, the "natural" units.

In the two schematic examples presented above, we could see that if the man-established quantities are replaced by the natural quantities, expressed in the natural units (with the help of Planck length and speed of light) then the fundamental constants, as the gravitational constant, permittivity of vacuum, and Planck constant, 
simply disappear from the equations describing a physical problem. This circumstance implies that these constants are, most probably, only the transformation constants between the artificial, man-established and natural quantities.

\section{Full-Sphere versus Hollow-Sphere Models}

In practice, every inward-proceeded part of the numerical integration of Equations (11)-(13), when it is started in a distance larger than zero, ends with the implication of the inner physical surface. (In principle, the numerical integration from a finite distance can end in the center with finite energy density and pressure. This is, however, only a single of infinite variety of possibilities. If there is no special attention to the choice of initial conditions, the probability of its occurrence approaches zero.) Such the property can be also expected for some other equations of state, not only the Chandrasekhar's Equation (7) and Equation (8). (Actually, the same qualitative behavior occurs using, e.g., the polytrope. And, the energy-density and pressure maximum in a finite distance also occurs for the equation state of radiation, $E=3 P$.) On the other-hand side, there is possible to construct a model of NS in the form of full sphere, if the integration starts in the center. (Then, we force the single of the infinite variety of possibilities to be the case.) In this section, let us reveal the conditions implying the full-sphere model of NS.

The realistic solution of the Equations (11)-(13) in the TOV problem has to satisfy the following demands. The pressure and energy density inside the object must acquire only finite positive values and have to decrease to zero at the object's physical surface. In the full-sphere model, these quantities must be finite in the center. The last requirement implies the asymptotic behavior $P \rightarrow a_{o}+a_{1} r+\cdots$ and $E \rightarrow b_{o}+b_{1} r+\cdots$ for $r \rightarrow 0$. $a_{o} \neq 0, a_{1}, b_{o} \neq 0$, and $b_{1}$ are the constant coefficients. Because the energy density approaches the constant $b_{o}$ for $r \rightarrow 0$, Equation (7) and Equation (12) yield $u \rightarrow \kappa b_{o} r^{3} / 6+C_{o}$ in this limit, where $C_{o}$ is an integration constant. If $C_{o} \neq 0$, then $\mathrm{d} P / \mathrm{d} r \rightarrow\left(a_{o}+b_{o}\right) /(2 r) \rightarrow \infty$ (see Equation (6)) for $r \rightarrow 0$. Therefore, it is necessary to demand $C_{o}=0$. Consequently, $\mathrm{d} P / \mathrm{d} r \rightarrow-\left(a_{o}+b_{o}\right) \kappa\left(a_{o} / 2+b_{o} / 6\right) r \rightarrow 0$ for $r \rightarrow 0$.

When $C_{o}=0$ and, therefore, $u \rightarrow \kappa b_{o} r^{3} / 6$, function $\exp (-\lambda)$, calculated from relation (5), approaches $\exp (-\lambda) \rightarrow 1-\left(\kappa b_{o} / 3\right) r^{2} \rightarrow 1$ for $r \rightarrow 0$. For $P \rightarrow a_{o}, E \rightarrow b_{o}$, and $\mathrm{d} P / \mathrm{d} r \rightarrow 0$, Equation (6) implies $v^{\prime} \rightarrow 0$ and, further, $v \rightarrow$ const. for $r \rightarrow 0$. This constant is unique for the object of given mass (implied by the given value of the maximum Fermi impulse, $p_{F, \max }$, in the object's center), because the metrics is continuous in the outer surface only for a single specific behavior of $v$. Hence, the $g_{r r}$ component of metric tensor converges to its value for the flat spacetime and $g_{t t}$ converges to a unique constant. So, the metrics of the full-sphere NS must obey $g_{r r} \rightarrow 1$ and $g_{t t} \rightarrow$ const. in the limit $r \rightarrow 0$.

We note that a difference between the full-sphere and hollow-sphere models can be practically negligible. In Figure 3, there is an example of such two similar models. In the hollow-sphere model, the distance of zero net gravity, $r_{o}$, is chosen in the distance of 1 meter from the center. In this distance, the input value of Fermi impulse is chosen to be the same as in the center of full-sphere model. We put it to equal to, e.g., $1.2 m_{n} c$. In Figure 3 , we can see that the models are practically identical from the outer radius of both spheres down to the star-centric distance $r \sim 10^{-8} \mathrm{~km}$. Of course, there can be created, in principle, a hollow-sphere model with an arbitrarily small $r_{o}$. The interval of similarity can be prolonged to even a shorter distance.

Since $r_{o}$ can be even smaller than a step of numerical integration, $\Delta r$, we can sometimes construct a model in form of a quasi full sphere. It is a hollow sphere, in fact, but one cannot recognize its true character because $r_{o}<\Delta r$.

We can conclude that the border between the traditionally unacceptable and acceptable models seems to be only formal: the model with exactly zero $r_{o}$ is acceptable, but almost identical model with a negligibly small, but finite $r_{o}$ is not longer acceptable in the traditional concept of NSs. One can, however, seriously doubt whether the hollow sphere model of NS is actually unacceptable and postulate P2 is an actually reasonable requirement for a realistic model of NS.

\section{A Wider Variety of the Ni's Models of Neutron Stars}

In this section, we introduce several sequences of the NS models constructed by using the Ni's solution of the TOV equations in course to map some properties of them. To obtain the models, we again start the numerical integration of Equations (11)-(13) in the distance of zero net gravity, $r_{o}$, where the initial value of function $u$ is given by relation (25). The values of $r_{o}$ are chosen to be $1 \mathrm{~m}, 1,5,10,15,20$, and $20 \mathrm{~km}$. For each value of $r_{o}$, 

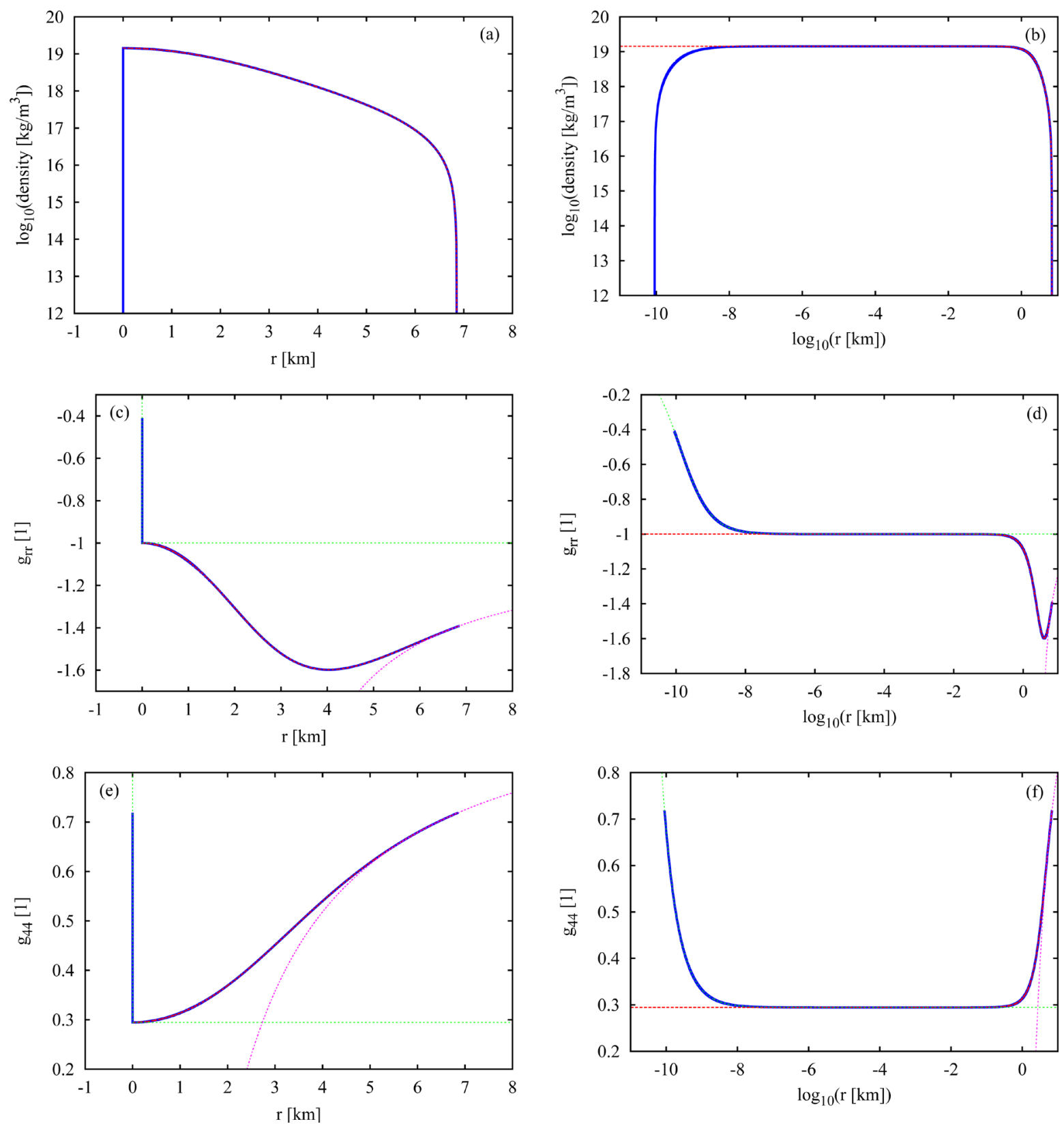

Figure 3. The comparison of almost identical TOV full-sphere and Ni's hollow-sphere models of NS. The top, middle, and bottom plots show the behavior of density, component $g_{r r}$, and components $g_{t t}$ of metric tensor, respectively. The left (right) plots show the behavior of the quantities in the linear (decadic-logarithm) scale of distance $r$. While the thick, solid, blue line shows the behavior of the quantities inside the NS body for the hollow-sphere model, the thick, dashed, red line does so for the full-sphere model. The thin, dotted lines touching the ends of the thick curves show the behavior of the components of metric tensor in the corresponding OSS, the violet (green) curve for NS-exterior vacuum in the outer (inner) space.

we integrate the sequence of models considering the series of input values of Fermi impulse from 0.2 to $2.4 m_{n} c$, with the step of $0.2 m_{n} c$. For each model, we perform an iteration to find the appropriate input value of $v$, which yields the continuous metrics at the outer physical surface of NS.

The result for some considered values of $r_{o}$ is illustrated in Figure 4. This figure shows the relation between the size of the object in given model and its gravitational mass. The pair of circles linked together by a solid line show the extent of the neutron object in given model. The square between these circles shows the position of the 

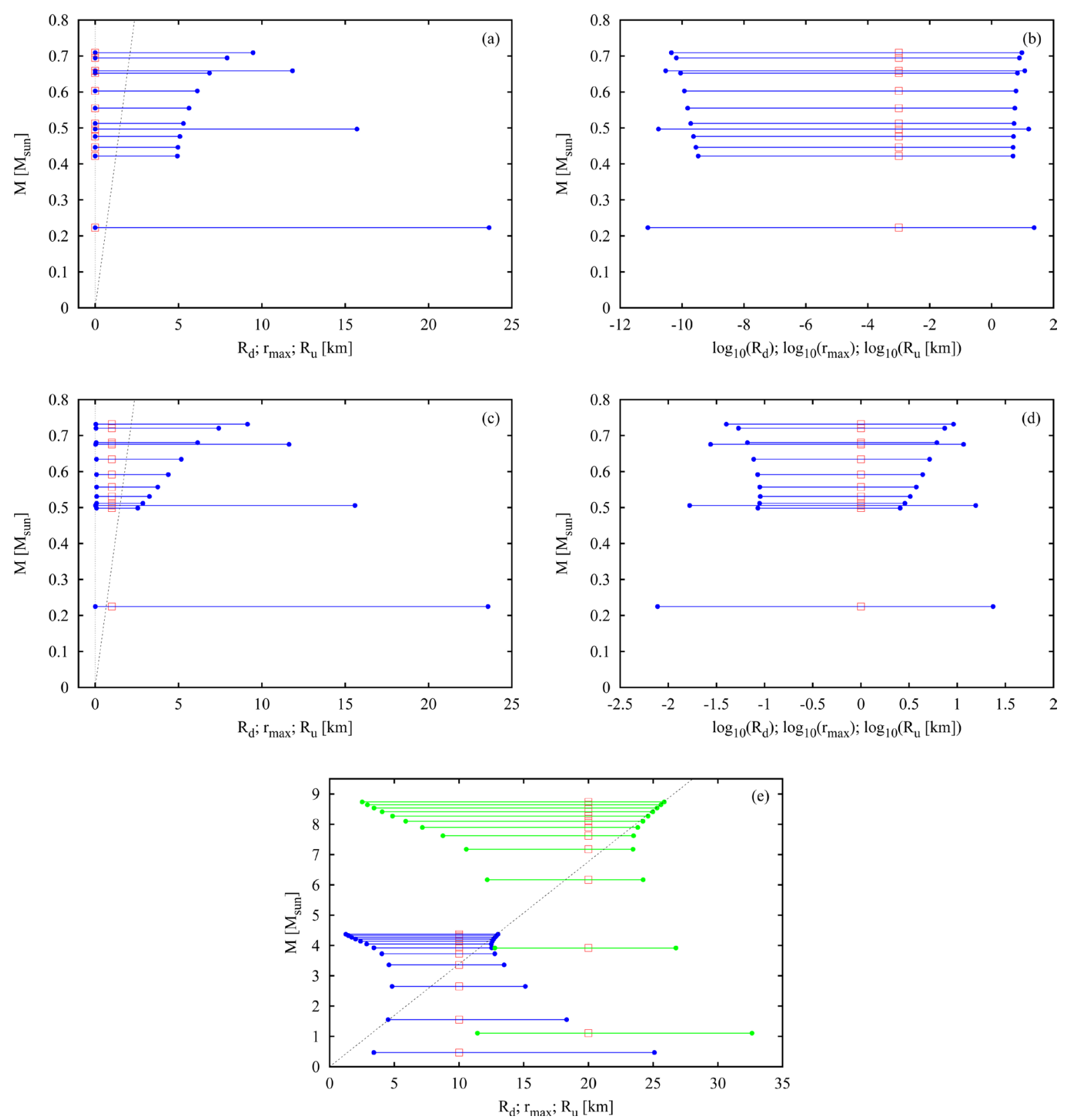

Figure 4. The size of NSs of various mass. Each pair of circles linked with a solid horizontal line shows the position of inner (left circle), $R_{i n}$, and outer (right circle), $R_{\text {out }}$, radius in the model of given mass, $M$. The square between both circles shows the radial distance $r_{o}$ with zero net gravity and maximum energy density and pressure. The dotted straight-line shows the behavior of the Schwarzschild gravitational radius. The plots (a) and (c) show the same models as plots (b) and (d), respectively, but the distance scale in plots (b) and (d) is logarithmic.

distance $r_{o}$, where the net gravity is zero and energy density and pressure are largest. The dotted straight-line shows the behavior of the Schwarzschild gravitational radius in the dependence on the mass.

For a relatively small value of $r_{o}$ (Figures $4(\mathrm{a})$-(d) for $r_{o}=1 \mathrm{~m}$ and $r_{o}=1 \mathrm{~km}$ ), the outer radius, $R_{\text {out }}$, monotonously decreases with increasing maximum Fermi impulse, $p_{F, \max }$. On the other-hand side, the mass is not any monotonous function of $p_{F, \max }$, but reaches a maximum and again decreases with increasing $p_{F \text {,max }}$.

For a relatively large value of $r_{o}$ (Figure 4(e) for $r_{o}=10$ and $20 \mathrm{~km}$; but it is found that the same is qualitatively also valid at least for $r_{o}=5,15$, and $25 \mathrm{~km}$ ), the outer (inner) radius decreases (increases) with in- 
creasing $p_{F, \max }$ at small value of $p_{F, \text { max }}$. For $p_{F, \text { max }}$-values above a critical value, the outer (inner) radius on contrary increases (decreases) with increasing $p_{F, \max }$. In Figure 4(e), one can see that the outer radius asymptotically approaches, from outside, to the Schwarzschild gravitational radius. We can expect that both radii become identical in the limit of $p_{F, \max } \rightarrow \infty$ and, consequently, in the limit of infinite internal energy of object.

Using the Ni's solution, it appears that there is a variety of NSs constituted by the same number of neutrons. In other words, we can construct a variety of models for a given rest mass, but with the different other characteristics. In Table 1, we present several such the models for the NS with the same rest mass as in the example shown in Figure 3. This rest mass is $0.6692 \mathrm{M}_{\odot}$. For lower-mass part of these models, there exist two models with the same distance $r_{o}$ (the second models are listed in the second part of Table 1) since two input values, $p_{F, \max }$, yielding the demanded rest mass can be found.

\section{On the Central Singularity}

In the past astrophysical applications of GR, there was well-known the existence of the true singularity in the center of black hole. Because this singularity was situated below the event horizon, it was regarded as having no concern to any observer residing in our universe, i.e. outside of the black hole. Because of this reason, there is the convention that this kind of singularity is not problematic and the metrics containing it can be accepted for a description of real objects. (The singularity is not "naked" singularity.)

On contrary, some solutions of the EFEs yield a true singularity that is not situated inside the event horizon and that can be, in principle, "experienced" by an observer. This kind of singularity is known as the "naked singularity" and its acceptability in a realistic description was ruled out by so-called "cosmic censorship" theorem [16]. According to the latter, no naked singularity, other than the Big-Bang singularity, can exist in the universe and, hence, in the theory, when all the considered conditions are realistic. We note, the naked singularity, other

Table 1. Some characteristics of the models of NSs consisting of the same number of neutrons, i.e. with the same rest mass, $M_{o}=0.6692 \mathrm{M}_{\odot}$. The models are created by using the Ni's solution of the EFEs. The explanation of symbols can be found in the text.

\begin{tabular}{|c|c|c|c|c|c|c|}
\hline$r_{o}[\mathrm{~km}]$ & $\begin{array}{c}p_{F, \max } \\
m_{n} c\end{array}$ & $\begin{array}{c}M \\
{\left[\mathrm{M}_{\odot}\right]}\end{array}$ & $u_{\text {out }}[\mathrm{m}]$ & $u_{i n}[\mathrm{~m}]$ & $R_{\text {in }}[\mathrm{km}]$ & $R_{\text {out }}[\mathrm{km}]$ \\
\hline \multicolumn{7}{|c|}{ PART 1} \\
\hline 0.001 & 0.58151 & 0.64931 & 958.932 & $-4.77401 \times 10^{-9}$ & $2.824 \times 10^{-11}$ & 12.119 \\
\hline 0.01 & 0.58151 & 0.64931 & 958.934 & $-4.77403 \times 10^{-6}$ & $2.824 \times 10^{-8}$ & 12.119 \\
\hline 0.1 & 0.58131 & 0.64930 & 958.918 & $-4.76779 \times 10^{-3}$ & $2.820 \times 10^{-5}$ & 12.119 \\
\hline 0.5 & 0.57527 & 0.64900 & 958.470 & -0.573147 & $3.4168 \times 10^{-3}$ & 12.159 \\
\hline 1. & 0.55279 & 0.64717 & 955.769 & -3.96729 & $2.4738 \times 10^{-2}$ & 12.391 \\
\hline 1.5 & 0.51908 & 0.64349 & 950.342 & -10.7004 & $7.2415 \times 10^{-2}$ & 12.838 \\
\hline 2. & 0.48311 & 0.63851 & 942.990 & -19.6935 & 0.14706 & 13.415 \\
\hline 3. & 0.41928 & 0.62671 & 925.558 & -40.5656 & 0.36929 & 14.727 \\
\hline 5. & 0.33025 & 0.60219 & 889.345 & -82.4425 & 1.0461 & 17.461 \\
\hline 10. & 0.21951 & 0.55351 & 817.452 & -161.948 & 3.5721 & 24.082 \\
\hline 15. & 0.16717 & 0.52114 & 769.649 & -213.078 & 6.7370 & 30.406 \\
\hline 20. & 0.13632 & 0.49853 & 736.251 & -248.191 & 10.245 & 36.541 \\
\hline 25. & 0.11583 & 0.48182 & 711.579 & -273.874 & 13.969 & 42.547 \\
\hline \multicolumn{7}{|c|}{ PART 2} \\
\hline 0.001 & 1.20000 & 0.65249 & 963.631 & $-6.46664 \times 10^{-8}$ & $8.981 \times 10^{-11}$ & 6.8558 \\
\hline 0.01 & 1.19999 & 0.65249 & 963.626 & $-6.46648 \times 10^{-5}$ & $8.981 \times 10^{-8}$ & 6.8558 \\
\hline 0.1 & 1.19846 & 0.65247 & 963.601 & $-6.43025 \times 10^{-2}$ & $8.926 \times 10^{-5}$ & 6.8538 \\
\hline 0.5 & 1.20478 & 0.64958 & 959.329 & -8.06360 & $1.0301 \times 10^{-2}$ & 6.6126 \\
\hline 1. & 1.51319 & 0.60928 & 899.815 & -144.763 & $8.1783 \times 10^{-2}$ & 4.7028 \\
\hline
\end{tabular}


than that of Big-Bang type, would cause a collapse of matter into a point in the center, in which the density would increase above all limits.

In the model of NS based on the Ni's solution, the metrics inside the internal cavity, which is bordered by the sphere of radius $R_{i n}$, is characterized with the metric tensor, the components $g_{r r}$ and $g_{t t}$ of which are given by relations (27) and (29). In the last relation, we see that $g_{t t} \rightarrow \infty$ when $r \rightarrow 0$. In this section, we discuss the basic properties of this central singularity, especially its concern to the naked singularity and cosmic censorship.

We note, there is not, either, the local Lorentz frame in the central point, with $r=0$, in the cavity.

It was empirically found that always $u_{i n}<0$. Hence, the denominator in relation (19) for $N_{\text {in }}$ is positive. As well, it was empirically found that $R_{\text {out }}>2 u_{\text {out }}$ and, thus, the nominator in relation (19), $1-2 u_{\text {out }} / R_{\text {out }}$, is also positive. Consequently, constant $N_{\text {in }}$ given by relation (19) is positive. Since the gravity is proportional to $\mathrm{e}^{-\lambda} v^{\prime}$, which coincidentally equals $\mathrm{e}^{-\lambda} v^{\prime}=\mathrm{e}^{v} v^{\prime}=\mathrm{d} g_{t t} / \mathrm{d} r$ in $r<R_{\text {in }}$ (as well as in $r>R_{\text {out }}$ ), it must be oriented outward within the sphere of radius $R_{i n}$ as seen after performing the derivative of $g_{t t}$ given by relation (29). (Its sign is opposite than that of the derivative of $g_{t t}$ for the outer empty space given by relation (28).) Thus, the central singularity is repulsive in the sense that every material object is attracted away from it. Maybe, this claim seems to be a paradox. We should however realize that a test particle in the region $r<R_{i n}$ is not repelled by the abstract singularity (an empty-vacuum-point in the NS center), but attracted by the circumambient real matter of NS, in fact. This attraction diverges in the limit $r \rightarrow 0$.

Since it is found that the outer radius is always larger than the Schwarzschild gravitational radius and all components of metric tensor are continuous (and everywhere finite) functions of radial distance in every distance $r>0$, the NS body including, there is no other than the central singularity. Because of this fact, the central singularity seems to be the "naked singularity". However, its repulsive character discriminates between it and the true, problematic, naked singularity. This singularity is the naked singularity, but that of Big-Bang type because of its repulsive character. It does not lead to the collapse of matter into any infinitesimally small volume and infinite increase of density. And, no material object can enter it.

Since the Ni's solution implies the models of compact objects without any singularity other than the above-mentioned Big-Bang type singularity, it enables us to accept a more strict than the original cosmic censorship. Specifically, we can demand that "no singularity other than the Big-Bang type singularity can exist in the universe and, hence, in the theory, when all the considered conditions are realistic". It means that adjective "naked" can be omitted in the theorem.

In conclusion, the main reason to postulate the Minkowski metrics in the vacuum inside a spherical shell is abolished in the Ni's concept of hollow sphere.

\section{Conclusion Remarks}

The GR was originally created as the geometric theory. Since the geometry of spacetime is determined by stress-energy tensor, the quantities as energy and (effective) gravitational potential are the integral part of the theory, except of the geometrical aspects.

Meanwhile, an alternative to the original intent of truly geometrical theory started to be used, especially in the theory of NSs: the concept of mass within the Newtonian concept of potential was regarded as the integral part of the GR. This concept of the potential differs from the approximation of the GR effective potential for a weak field by an absence of constant term $c^{2}$. The demand of the validity of the Newtonian concept implies that the size of $g_{r r}$-component of metric tensor in the TOV problem must always be larger than unity. As a consequence of this constraint, there is no stable solution of the EFEs for an object with mass above the Oppenheimer-Volkoff limit. And, we can obtain only the solution of the EFEs with the monotonous, everywhere inward oriented gravity.

Consequently, the traditional model of spherically symmetric NS, as firstly found by Oppenheimer and Volkoff [1], is only the single of infinite variety of realistic NS models in GR. A further, infinite set of models appears when we permit that the size of the $g_{r r}$-component of metric tensor is also lower than unity. The essential effect corresponding to this permission is the outward oriented net gravitational attraction of upper stellar layers (material being in a larger distance from the center than a given test particle).

In the Euclidean space of Newtonian physics, the net gravity of upper layers is proved to be exactly zero in the case of spherical symmetry. However, the conception of spherical symmetry in the curved spacetime of GR 
is more complicated. A concentric layer, which is observed as spherically symmetric by the observer in the stellar center, is not longer observed, in general, as spherically symmetric by an observer aside the center. Hence, its gravitational action on a particle situated inside the layer but aside its center is generally non-zero. The solution of the EFEs found by $\mathrm{Ni}$ [3] implies that it is actually finite and oriented outward from the center.

Inside the NS, the outward oriented net gravity of upper layers increases with the decreasing radial distance, since the mass of upper layers increases. The behavior of net gravity of lower layers is opposite, of course. In the NS's interior, there is a critical distance in which the partial gravitational actions of both upper and lower layers equal each other. Below this distance, the net gravity of upper layers becomes dominant and, thus, the total net gravity is oriented outward. In the stable configuration, this gravity is again balanced by the gradient of pressure, which is always oriented against the gravity, according to the equation for the gradient derived from the EFEs. The inner physical surface is formed by the same mechanism as the outer surface.

So, the NS model constructed by using the Ni's solution of the EFEs is the hollow sphere with a cavity in its interior. The existence of this cavity is enabled by the fact that the metrics inside it is again described by the OSS, but with sign plus in front of the fraction $2|u| / r$ figuring in the formulas for the components of metric tensor. It means that the corresponding quantity $u$ is negative. Thus, the gravitational attraction in the cavity is oriented outward. Because of this orientation, there is no problem with the naked, but of Big-Bang type central singularity. And, there is no reason to reject the application of the Ni solution of EFEs in the astrophysics of real for-central-observer spherically symmetric objects.

The negative values of $u$ become physically acceptable, when this quantity is no longer represented in the term of Newtonian-type gravitational potential, but is simply regarded as the metric quantity (alternative form of $\left.g_{r r}\right)$. This requires ruling out "mass" as the regular physical quantity from the GR. In gauging of the integration constants yielded by integrations of the EFEs with the help of Newtonian physics, it is then necessary to strictly keep the different meanings of $u$ and mass. In a weak field and region of radial distances $r>R_{\text {out }}$, where always $u=u_{\text {out }}>0$, the correspondence between this parameter and $G M / c^{2}$ can be supposed, but we should not inversely consider mass $M$ within the GR, which is intended to be the purely geometrical theory. (We demonstrated that the EFEs in the TOV problem can be re-written to the dimensionless form. Most likely, this is possible generally. The concept of mass then becomes useless.) And, we should not further generalize the concept of mass and use it in any argumentation within the GR.

The concept of hollow sphere is able, as already claimed by Ni [3], to create a model of stable compact object of whatever large mass. Such that the conclusion can seem to be in a contradiction with that by Oppenheimer and Snyder [2] who described the collapse of every compact object with a mass larger than a certain upper limit. As well, it contradicts to the conclusion published by Rhoades and Ruffini [17] about the maximum mass of NSs. These works were, however, implicitly based on postulate that the total net gravity must be oriented inward inside the whole NS. Thus, they are valid only for the full-sphere concept of NS.

With the concept of hollow sphere, we can moreover avoid any singularity, other than the central Big-Bang type singularity, in the astrophysics of real objects. Thus, it seems that we can eliminate several serious problems in the current astrophysics with the help of the Ni's solution of EFEs.

\section{Acknowledgements}

The work was supported, in part, by the VEGA — the Slovak Grant Agency for Science, grant No. 2/0031/14, and by the Slovak Research and Development Agency under the contract No. APVV-0158-11.

\section{References}

[1] Oppenheimer, J.R. and Volkoff, G.M. (1939) Physical Review, 55, 374-381. http://dx.doi.org/10.1103/PhysRev.55.374

[2] Oppenheimer, J.R. and Snyder, H. (1939) Physical Review, 56, 455-459. http://dx.doi.org/10.1103/PhysRev.56.455

[3] Ni, J. (2011) Science China: Physics, Mechanics, and Astronomy, 54, 1304-1308. http://dx.doi.org/10.1007/s11433-011-4350-9

[4] Tolman, R.C. (1939) Physical Review, 55, 364-373. http://dx.doi.org/10.1103/PhysRev.55.364

[5] Chandrasekhar, S. (1935) Monthly Notices of the Royal Astronomical Society, 95, 207-255. http://dx.doi.org/10.1093/mnras/95.3.207

[6] Landau, L. (1932) Physikalische zeitschrift der Sowjetunion, 1, 285. 
[7] Einstein, A. (1915) Sitzungsberichte der Preussischen Akademie der Wissenschaften zu Berlin, 844-846.

[8] Einstein, A. (1916) Annalen der Physik, 354, 769-822. http://dx.doi.org/10.1002/andp.19163540702

[9] Eddington, A.S. (1922) Relativitätstheorie in Mathematisher Behandlung. Alexander Ostrowski and Harry Schmidt, Göttingen and Cöthen. (In German)

[10] Birkhoff, G.D. and Langer, R.E. (1923) Relativity and Modern Physik. Harvard University Press, Cambridge, Mass.

[11] Schwarzschild, K. (1916) Sitzungsberichte der Königlich Preussischen Akademie der Wissenschaften zu Berlin, Phys.Math. Classe, 1, 189-196.

[12] Misner, C.W., Thorne, K.S., and Wheeler, J.A. (1997) Gravitation. 20th Printing, W. H. Freeman et Comp., New York.

[13] Tolman, R.C. (1969) Relativity, Thermodynamics, and Cosmology. Clarendon Press, Oxford.

[14] de Broglie, L. (1925) Annales de Physique, 3, 22.

[15] de Broglie, L. (1925) Comptes Rendus de l'Académie des Sciences, 180, 498.

[16] Penrose, R. (1969) Nuovo Cimento, 1, 252-276.

[17] Rhoades, C.E. and Ruffini, R. (1972) Physical Review Letters, 32, 324-327. http://dx.doi.org/10.1103/PhysRevLett.32.324 